\title{
STUDY ON THE EVOLUTION OF THE ROMANIAN WOMEN'S EPEE TEAM AT THE OLYMPIC GAMES
}

\author{
Amalia Alexandra TĂTĂRAN ${ }^{1 *}$, Silvia TEODORESCU ${ }^{1}$, Gabriela DINȚICĂ ${ }^{1}$ \\ ${ }^{1}$ National University of Physical Education and Sport, Faculty of Physical Education and Sport, Bucharest, \\ Romania \\ *Corresponding author: amalia.tataran@yahoo.com
}

DOI: https://doi.org/10.51267/icpesk2020bp16

\begin{abstract}
This study refers to the evolution of the Romanian Women's Epee Team at the Olympic Games in terms of performance, but especially from the perspective of making up the team and establishing the strategy for addressing each goal-focused competition. The literature review reveals that one of the ways to increase both the quality of the training process in fencing and the success rate in team events lies in the team composition, but also in establishing the sequence of athletes competing in a match. Research purpose: The study aims to highlight the dynamics of the Romanian Women's Epee Team through both a detailed analysis of the results achieved by athletes in their fight against various opponents and their comparison at different times of the matches. Methods: Bibliographic study, comparison, abstraction, induction and deduction methods, video analysis, mathematical and statistical methods. Results and conclusion: The sequence of athletes' performance in team competitions and their effectiveness depending on the number of won/lost bouts, but also successful hits and recovery times were analysed based on the competition protocols for the Rio de Janeiro and London Olympics, and the performance of the Romanian Women's Epee Team was compared with the performance achieved by other competing teams. The study has shown that, in today's modern fencing (particularly the Epee), one of the most important factors for achieving performance in the team event refers to making up the team so that it can work as a whole in relation to the opposing team's composition.
\end{abstract}

Keywords: fencing, team competitions, Epee, individual performance, Olympic Games, tactics.

\section{Introductiom}

The world of weapons has been dominated by men ever since the knights were fighting on the battlefield, the presence of women in such exploits being a rarity in the past. In modern fencing competitions, three weapons have been established: foil, epee and sabre. Fencing was included into the programme of the Olympic Games from their first edition, but originally it was exclusively a male sport. Women's fencing was accepted into the Olympic programme at the 1924 Paris Summer Games before many other sports, as a result of an ambiguity in the regulations the International Olympic Committee (IOC) and the International Fencing Federation (FIE). The full integration of female fencing took more or less, according to the various weapons used in competitions. Thus, the individual foil event appeared in 1924, the team foil event was not recognised until 1960, and the other weapons were integrated even later. The female Epee became an Olympic event in 1996, and the female Sabre, in 2004, after long debates both within the FIE and between the FIE and the IOC. (Terret \& OttogalliMazzacavallo, 2012)

As regards the medals won by the Romanian fencers from the beginning of the modern Olympics and until now, a number 14 medals can be counted (Dinţică, 2018). At the 1996 and 2000 Olympics (where only the individual event took place), our country was not represented in the female Epee event, which is actually the topic of our study. 
Fencing is a sport performed in a gym where two athletes fight with their weapons, the close combat (body contact) being prohibited. The fight is called a bout, and the point is called a hit (touche). Fencing takes place on a $14 \times 1.5 / 2-\mathrm{m}$ strip (or piste). The fight is mediated by a referee, and the hits are monitored by an electric device. For the Epee event, the device was introduced in 1936, for the Foil event - in 1956, and for the Sabre event - in 1988 (Roi \& Bianchedi, 2008). In individual events, the winner is the first fencer to score 5 hits during the preliminary pools, or 15 hits during the elimination bouts. During the preliminary pools, bouts last for 5 minutes, while during elimination, each bout consists of 3 rounds of 3 minutes, with 1-minute rest between them. If an athlete fails to score 5 hits within the 3 minutes, the winner is the fencer who has an advantage before the time is up. If the score is tie, 1 more minute of fight is granted, and the bout will be won by the fencer who succeeds in scoring a golden hit or has priority awarded by the device before the minute starts. In team events, the match is played up to 45 hits ( 9 bouts of 5 hits each for 3 minutes at the most), or the winner will be the leading team at the end of the 9 bouts. If the score is tie when the time for the last bout is up, the same rule is used as in the individual event. The team event involves 3 athletes and 1 substitute fencer. Matches are played as follows: each athlete of a team against each athlete from the opposing team, in a sequence preestablished by the regulation. During a competitive bout, 5 hits or less can be scored. If a fencer scores less than 5 hits, the next teammate can recover the hits missed by the previous player. The substitute athlete can replace anyone in the team if the change is announced before the previous bout. (Turner et al., 2014)

Fencing tournaments take place over a whole day (often lasting about 10 hours) and consist of approximately 10 bouts with a break of around 15 to 300 minutes between each bout (Roi \& Bianchedi, 2008).

Fencing is characterised by the struggle between the tactical intentions of the two athletes, and the accuracy and insight of thinking, but especially creativity, play an important role in winning the victory. Fencing is an indoor sport that requires specific equipment, specialised coaches and hard work. Due to its specificity, direct fight against the opponent has a deep psychological character, all the more that the victory does not belong to the athlete who has better developed muscles, but to the athlete who possesses superior intelligence.

Fencing involves maximal effort performed with intermittence, meaning that athletes experience rapid alternation between resting and intensive activity during a match (Murgu, 2006).

The dominant abilities of the armed arm, in physical terms, are agility and speed (of reaction and execution) with a high degree of complexity. The variable conditions of competitive activity in fencing establish the priority in the training structure of skilled athletes and the effectiveness of technical and tactical training (Briskin et al., 2016).

Fencing is often considered a physical game of chess. Fencing bouts require a multitude of offensive and defensive movements, but also the special mental agility of "reading" the opponent's thinking and combating it. They often need to happen in the same time or be separated by only few seconds. The dominant abilities of the leg movements are endurancestrength, endurance-speed and mobility.

Nowadays, fencing development requires improving the various training components in the case of performance athletes. It is obvious that coaches should apply the most effective 
and evidence-based scientific recommendations for increasing athletic performance and skills. (Ashmarin, 1978; Tyshler, 1997; Platonov, 2004, cited in Zadorozhna et al., 2018a) Current fencing trends indicate that a team's success depends on several methods and characteristics of technical, tactical and theoretical training sessions. The organisation of fencing team competitions creates the prerequisites for implementing the athlete's mental and physical abilities as part of the team; this is the only way to achieve remarkable results. (Diaz, 1984; Tyshler, 1997, cited by Zadorozhna et al., 2018a) However, the specificity of team competitions involves solving important tasks such as the rational sequence of athletes, or predicting and simulating matches in general, but particularly matches against certain opponents. Also, inadequacy of tactical decisions eliminates possible advantages over the opponent, and, in the presence of tactical faults, this may lead to defeat. (Zadorozhna et al., 2018a)

In the key moments of competitions, athletes experience various emotional states that can facilitate or, on the contrary, block the achievement of high sports performance. For female athletes, it has been found that perceived stress and negative emotions, at a moderate level and in the short term, lead to better data storage and processing ability (Predoiu et al., 2017).

A rational and scientifically grounded training system involves gaining knowledge and skills that can help athletes to solve the tasks of different training components that are closely interrelated (Matveev, 2001, cited in Zadorozhna et al., 2018b). Therefore, the initial link for improving any aspect of training consists in mastering special theoretical knowledge that determines not only an athlete's ability to perform operational analyses during competitive activities, but also their desire to independently manage the training process (Buylina \& Kuramshina, 1980, cited in Zadorozhna et al., 2018b).

At the same time, specialists from many countries agree that sports training is a holistic and multi-annual process during which the athlete aims at achieving top performance in order to fight in the most prestigious competitions. Reaching this goal is impossible without taking into account the fundamental principles of periodisation, which is understood as structuring of the training process and competition participation in different phases, periods and cycles. (Lyakh et al., 2016)

In this context, the performance route to the world's fencing elite in the Epee event has started for Romania in 2004, in Athens, when Ana Maria Brânză managed to qualify and was ranked 16th. At the Beijing 2008 Olympics, the same Ana Maria Brânză managed to rank 2nd in the individual Epee event (the team event did not take place). At the London 2012 Olympics, Romanian fencing had two athletes in the individual event, namely Anca Măroiu (5th place) and Simona Gherman (6th place), who were among the first 8 finalists, as well as Ana Maria Brânză (11th place). In the team event, Romania was ranked 6th, although it was a favourite for winning a medal (it was number 1 on the board before the start of the competition). At the Rio de Janeiro 2016 Olympics, the Romanian Women's Epee Team took revenge and won the gold medal. 


\section{Research purpose}

The study aims to highlight the dynamics of the Romanian Women's Epee Team through both a detailed analysis of the results achieved by athletes in their fight against various opponents and their comparison at different times of the matches.

\section{Methodology}

The research methods used were: bibliographic study, comparison, abstraction, induction and deduction methods, video analysis, mathematical and statistical methods.

Observation and pedagogical analysis of competition protocols for the London and Rio de Janeiro Olympics were mainly used. Mathematical calculation methods were also used to assess the indicators of athletes' individual performance. Thus, the total number of scored and received hits was calculated, and the (positive or negative) difference between them was considered an indicator of individual performance (effectiveness). These indicators were determined based on the individual performance of the participant in team matches played during the Olympic Games. It should be mentioned that the best participant was considered to be the team's number 1, having the greatest responsibility for the overall team result. Also, the team's number 3 was considered a less effective athlete.

\section{Results}

This study aimed to analyse the matches of the Romanian team against the teams of Germany, Italy and Korea at the London Olympics, as well as against the teams of the United States, Russia and China at the Rio de Janeiro Olympics.

During the research, the sequence of athletes' performance and their effectiveness in team competitions were also analysed. To this purpose, the competition protocols for the London and Rio de Janeiro Olympics were used.

At the London Olympic Games, the Romanian route was as follows: Romania - Korea (38-45), Romania - Italy (45-38), Romania - Germany (36-42) (Table 1).

Table 1. Romanian fencing performance at the London Olympics

\begin{tabular}{lcccccccccc}
\hline Athlete & \multicolumn{3}{c}{ Scored hits } & \multicolumn{3}{c}{ Received hits } & & Match index & $\begin{array}{c}\text { Overall } \\
\text { index }\end{array}$ \\
\hline & Korea & Italy & Germany & Korea & Italy & Germany & Korea & Italy & Germany & \\
GS & 18 & 15 & 13 & 19 & 12 & 15 & -1 & 3 & -2 & 0 \\
BA & 11 & 15 & 9 & 15 & 13 & 10 & -4 & 2 & -1 & -3 \\
MA & 9 & 14 & 14 & 11 & 13 & 15 & -2 & 1 & -1 & -1 \\
DL (BA) & 0 & 0 & 0 & 0 & 0 & 2 & 0 & 0 & -2 & -2 \\
\hline
\end{tabular}

Analysing the athletes' individual indices, we can say that their sequence in terms of effectiveness is: first position - athlete GS, second position - athlete MA, third position athlete BA, and fourth position - substitute athlete DL, who competed only in the match 3 , in a single bout. In general, a match ends with the athlete considered number 1 by the coach, the 
second-to-last bout - with the athlete considered number 2, and the bout preceding it - with the athlete considered number 3.

In order to establish the effectiveness of the technical and tactical plan for our athletes in relation to the opponents, we calculated the Spearman non-parametric correlation coefficient (by ranks) between scored hits and received hits (attack/defence) and between scored/ received hits and match index (final result) (Table 2).

Table 2. Values of Spearman correlation coefficient - London Olympics

\begin{tabular}{ccccccccc}
\hline \multicolumn{2}{c}{ Spearman coefficient } & \multicolumn{2}{c}{ Spearman coefficient } & \multicolumn{3}{c}{ Spearman coefficient } \\
Korea & & \multicolumn{3}{c}{ Germany } \\
\hline Scored/ & Match/ & Match/ & Scored/ & Match/ & Match/ & Scored/ & Match/ & Match/ \\
Received & Scored & Received & Received & Scored & Received & Received & Scored & Received \\
hits & hits & hits & hits & hits & hits & hits & hits & hits \\
1 & -0.4 & -0.4 & 0.55 & 0.95 & 0.316 & 0.95 & 0.447 & 0.235 \\
\hline
\end{tabular}

As can be seen, the strongest connection between scored and received hits was recorded in the match with Korea ( $\mathrm{r}=1)$, where unfortunately the result was not in favour of the Romanian team. The same good correlation $(r=0.95)$ is found in the match with Germany, where Romania also recorded a defeat.

A good correlation $(r=0.95)$ between scored hits and match index was found in the match with the Italian team, which was won by the Romanian athletes. We can conclude that an attacking strategy based on a high number of hits ensures victory in the competition.

At the Rio de Janeiro Olympics, the matches were as follows: Romania - US (24-23), Romania - Russia (45-38), Romania - China (44-38) (Table 3).

Table 3. Romanian fencing performance at the Rio de Janeiro Olympics

\begin{tabular}{lcccccccccc}
\hline \multicolumn{1}{c}{ Athlete } & \multicolumn{3}{c}{ Scored hits } & \multicolumn{3}{c}{ Received hits } & \multicolumn{3}{c}{ Match index } & Overall index \\
\hline & US & Russia & China & US & Russia & China & US & Russia & China & +8 \\
BA (PA) & 11 & 12 & 19 & 9 & 12 & 13 & 2 & 0 & 6 & +1 \\
GS & 5 & 13 & 17 & 4 & 6 & 16 & 1 & 7 & 1 & +9 \\
PS & 8 & 20 & 5 & 10 & 13 & 7 & -2 & 7 & -2 & +3 \\
DL (PS) & 0 & 0 & 0 & 0 & 0 & 2 & 0 & 0 & 1 & +1 \\
\hline
\end{tabular}

The analysis of the ratio between scored hits and received hits showed that athlete number 1 was GS, athlete number 2 - PA, athlete number 3 - PS, and the fourth athlete was DL, who competed only in the match 3 , in a single bout.

Table 4. Values of Spearman correlation coefficient - Rio de Janeiro Olympics

\begin{tabular}{ccccccccc}
\hline \multicolumn{2}{c}{ Spearman coefficient } & \multicolumn{3}{c}{ Spearman coefficient } & \multicolumn{3}{c}{ Spearman coefficient } \\
Cussia & \multicolumn{3}{c}{ China } \\
\hline Scored/ & Match/ & Match/ & Scored/ & Match/ & Match/ & Scored/ & Match/ & Match/ \\
Received & Scored & Received & Received & Scored & Received & Received & Scored & Received \\
hits & hits & hits & hits & hits & hits & hits & hits & hits \\
0.8 & 0.4 & -0.2 & 0.8 & 0.894 & 0.447 & 0.8 & 0.632 & 0.316 \\
\hline
\end{tabular}


Regarding the correlation between scored hits and received hits, it can be noted that the correlation coefficient has the same value, $\mathrm{r}=0.8$, in all three matches, which highlights a close association between these variables during the three matches that the Romanian female team managed to win. As for the correlation between scored hits and final result, very high and high values, $r=0.894$ and $r=0.632$, were recorded in the matches with Russia and China, which the Romanian team won with the scores 54-38 and 44-38, respectively. (Table 4)

Table 5. Values of Spearman correlation coefficient recorded at the two editions of the Olympics

\begin{tabular}{ccccccccc}
\hline \multicolumn{3}{c}{$\begin{array}{c}\text { Spearman coefficient } \\
\text { London and Rio Olympics } \\
\text { Match 1 }\end{array}$} & \multicolumn{2}{c}{$\begin{array}{c}\text { Spearman coefficient } \\
\text { London and Rio Olympics } \\
\text { Match 2 }\end{array}$} & \multicolumn{3}{c}{$\begin{array}{c}\text { Spearman coefficient } \\
\text { London and Rio Olympics } \\
\text { Match 3 }\end{array}$} \\
\hline Scored/ & Match/ & Match/ & Scored/ & Match/ & Match/ & Scored/ & Match/ & Match/ \\
Received & Scored & Received & Received & Scored & Received & Received & Scored & Received \\
hits & hits & hits & hits & hits & hits & hits & hits & hits \\
0.901 & -0.629 & -0.630 & 0.844 & 0.782 & 0.450 & 0.848 & 0.528 & 0.225 \\
\hline
\end{tabular}

Analysing the Romanian team's behaviour at the two editions of the Olympics (London 2012 and Rio de Janeiro - 2016) in terms of scored/received hits, the existence of a strongly positive correlation in all six matches is highlighted, and this result can be explained by the high level of training of the finalist teams in the two editions.

Regarding the correlation between scored hits and final score, it can be noted that the association values are increased only in match $2(\mathrm{r}=0.782)$, the Romanian team winning both matches with the same score (45-38) against Italy and Russia. Our rank correlation coefficient is 0.78 and we find that the significance threshold is lower than $p=0.05$, so we can say that there is a strong connection between the two variables. (Table 5)

The data analysed and processed by us do not emphasise a correlation between the number of received hits and the match index in any of the matches played at the two editions of the Olympics, which leads us to state that offensive behaviour is needed to win the victory.

\section{Discussion}

Specialists in the field (such as Zadorozhna et al., 2018a) consider that:

1. The specificity of fencing team competitions requires the definition of a rational tactical scheme, namely the sequence of athletes' performance during a team match. The basis for such a sequence should be the individual performance indices of athletes in a certain position during fights against different opposing teams.

2. The optimal sequence of athletes' performance in team competitions should be organised on the basis of individual performance indices of athletes in different positions: • the national team of Ukraine: first-number position - Kryvytska O., second-number position Panteleyeva K., third-number position - Bezhura F. and Pochkalova A.; • the national team of Estonia: first-number position - Embrich Irina, second-number position - Beljiaeva Julia, third-number position - Kuusk Kristin; - the national team of China: first-number position - 
Sun Yiwen, second-number position - Xiang Yixuan, third-number position - Zhu Mingye; • the national team of Korea: first-number position - Kong Man Wai Vivien, second-number position - Choi Injeong and Choi Eunsook, third-number position - Kang Young Mi.

3. Theoretically, it is considered that the team changes the second- and third-number positions depending on the level of competition, team evaluation and composition of opposing team, but the first-number position remains unchanged (Zadorozhna et al., 2018a).

It was not the same with the Romanian team that made some major changes during the competition: number 1 - BA (PA), considered to be number 1, was the second to last in the match with Russia, where athlete number 2 - GS ended the match (a beneficial tactical change, in our case). Changes in important moments can modify the match result, as it was in this case.

In the current stage of fencing development, the following approaches are used to form a team: team composition is stable during the season; all participants compete in a predetermined sequence; team composition is stable throughout the year, and the number/ position varies depending on the level of competition, team ranking and composition of the opposing team (Zadorozhna et al., 2018a).

The study aimed to analyse the sequence of athletes' performance in team competitions and their effectiveness depending on the number of won/lost bouts, successful hits and recovery times based on the competition protocols for the Rio de Janeiro and London Olympics, and also to compare the performance of the Romanian Women's Epee Team with the performance achieved by other competing teams.

The countries participating in the two editions of the Olympics were: London, $2012-$ China, Korea, USA, Russia, Germany, Romania, Italy and Ukraine; Rio de Janeiro, 2016 Romania, China, Russia, Estonia, USA, Korea, France, Ukraine and Brazil. As can be seen, two powerful fencing teams did not participate in Rio de Janeiro (Germany and Italy), being replaced by other countries with a tradition in fencing: France and Brazil. If the Romanian team started as the number 1 favourite at the London Olympics, at the Rio de Janeiro edition, it started from the second position.

The Romanian team did not compete against the same teams at the two editions of the Olympic Games.

In London, the matches were as follows: Romania - Korea (38-45), Romania - Italy (4538), Romania - Germany (36-42).

In the first match against the Korean team to qualify for the semi-finals, all our athletes had a negative index (GS -1, BA -4, MA -2), losing the match with -7. An unexpected score, given that our team had started from the favourite position. The match was unbalanced starting with the second bout, when the Korean team took the lead until the end of the match. The match against Italy was won with +6 , the best index, namely +3 , belonging to athlete GS, who demonstrated thus her number-one position in the team.

In the match for the 5th place, all athletes had again a negative overall index, -6 . But this time, the team's number 1 was the one who recorded the lowest index, -2 , losing the fifth bout with 6-1. In our opinion, this was a key moment because her teammates failed to keep up with the match from that point on. We believed that changing this athlete with the substitute fencer would have been beneficial, so athlete BA was changed. However, the substitute fencer failed to bring positive points to her team, and thus the match was lost, the 
team ranking 6th at the end of the competition. Athlete GS, although she had the best index in all three matches, lost bouts in key moments, when the team was counting on her. The match was won by China against the Korean team, which had started from the 8th place at the beginning of the competition.

In Rio de Janeiro, the matches were as follows: Romania - USA (24-23), Romania -Russia (45-38), Romania - China (44-38).

Between the two editions, team composition was modified by changing a single athlete, M.A, who was replaced by PS. Athlete BA is the same as PA, which is why we will name her BA (PA).

The match considered to be the most balanced was the first one, with the Unites States, where the Romanian team won very difficultly, perhaps also due to the pressure generated by the failure at the London Olympics. The score was favourable to our team throughout the match due to the special contribution of athlete BA (PA), who had the index +2 (one hit less than GS). It was a rather tactical match, where two bouts ended with the score $0-0$, and the last one, with the score 8-8, our team winning the victory because it had the advantage of one extra hit. The semi-final match was again led by the Romanian team from the beginning to the end. In this match, the team's number 1 was replaced by number 2, who closed the match. This change might have been the key to winning the match, the decision relying on the history of other competitions. In this match, it was noted that the athlete who used to be number 1 had the index 0 for scored/ received hits, while each of her teammates had the index +7 . This demonstrates that the coaches' experience played an important role when they decided to change the sequence of athletes competing in the match. The Olympics final was won against China with the score 44-38 without any problem. The closest score was in the fourth bout, when Romania was leading by a single hit (10-9). From that moment on, our team had an advantage of at least 3 hits over the opposing team. The substitute athlete DL was introduced in the seventh bout and managed to keep the advantage. In all three matches, the team athletes proved that they could complement each other very well and even recover and keep the score if necessary.

We noticed that, at the London Olympics, the sequence of athletes was the same in all matches, while at the la Rio de Janeiro Olympics, this sequence was changed in the second match, which was not ended by the same athlete. As for Romania, the team's number 1, PA (BA), closed the first and last matches.

Regarding the individual index of athletes, some observations can be made:

- the athlete considered the team's number 1 at the London Olympics (GS) kept her status, achieving an index of scored/received hits equal to 0 .

- the athlete considered the team's number 2 at the London Olympics did not have the expected effectiveness, recording a poorer index (-3) than athlete number $3(-1)$. In the third match, athlete BA was replaced by the substitute fencer in the last bout at the score 33-27 for Germany in the hope of improving the score, but this did not happen.

- At the Rio de Janeiro Olympics, athlete GS was considered number 2, but her overall index showed that she had the best team index.

Given that the matches had a fairly tie score, the final being at a difference of 6-7 hits, we can consider that the formula chosen at that moment was not the best one since the athlete ranked 2nd in terms of effectiveness during the last match (with Germany) was changed. 
Compared to London, at the Rio de Janeiro Olympics, the sequence of athletes competing in the second match (with Russia) was changed, and thus athlete GS (considered to be the team's number 2) ended the match. This was beneficial because the opposing team was sufficiently destabilised to lose the match in favour of Romania.

From the index obtained by each athlete, it can be noticed that this time too GS was number 1 with the index +9 , compared to the next athlete, BA (PA), whose index was +8 .

We believe that the close value of the team athletes and the changes made in key moments were a beneficial factor in winning the Olympic title. Another important thing is that, at the London Olympics, athletes had a negative index or an index equal to 0 (GS), while at the Rio de Janeiro Olympics, all athletes had a positive overall index.

Another observation is that the matches played in London ended with 45 hits ( 2 matches) and 42 hits (1 match). At the Rio edition, the first match was won by our team with the score 24-23 (priority), its highly tactical character being also noted. The other two matches, although considered more difficult, went up to 45 and 44 hits, respectively.

The index between the two editions was -6 in London (6th place) and +21 in Rio de Janeiro (1st place). The Romanian team participated in the best sports competitions in the world throughout more than two Olympic cycles, which highlights that it is a homogeneous, mature and well-trained team. The fact that our team kept the core of the previous team was an important step in winning the supreme title. To achieve great performance in the Epee team event after the London Olympics involved reconsidering the training process in its whole by combining strategies focused on the scientific management of physical effort with the psychological and methodological assistance for athletes in order to turn the traininginduced technical and tactical gains into better results. In our case, performance training is a management tool used to achieve maximum results in competitions through fundamental changes in the attitude and behaviour of athletes, determined by their motivation and actionrelated expectations.

\section{Conclusion}

The current study highlights the following aspects:

In Women's Epee - team event, the final result achieved in a major competition is influenced by the athlete's individual behaviour in each match, but the determining factor is the offensive tactical behaviour of the entire team (the Spearman correlation coefficient between scored hits and match index has values in the range $[r=0.782-0.95]$ ).

Good individual team ranking does not guarantee winning a team medal. Although, at the London Olympics, the Romanian team athletes were individually ranked 5th, 6th and 11th, they did not manage to surpass other countries that had one or maximum two athletes among the first 16 (Korea - 4th and 12th places, China - 3th and 9th places). In the individual competition at the Rio de Janeiro Olympics, Romania was ranked 9th - BA(PA), 16th - GS and 33rd - PS, while China was ranked 3rd, 17th and 21st, the other medals being shared by athletes from Hungary and Italy.

A team with good competitive experience provided by its participation in the most important sports events at world level, which is well trained and homogeneous in terms of 
value level of the components can successfully manage tactical situations imposed by opponents.

The study has shown that, in today's modern fencing (particularly the Epee), one of the most important factors for achieving performance in the team event refers to making up the team so that it can work as a whole in relation to the opposing team's composition.

\section{Authors' Contributions}

All authors have equally contributed to this study and should be considered as main authors.

\section{References}

Briskin, Y., Semeryak, Z., Pityn, M., \& Vaulin, O. (2016). Qualified women epee fencers technical and tactical training using of highly qualified sportsmen model indicators. Journal of Physical Education and Sport, 16(2), 534-539. DOI: 10.7752/jpes.2016.02084

Dințică, G. (2018). Romanian fencing, a constant presence at the Olympic Games. European Proceedings of Social and Behavioural Sciences, 36, 18-28. http://dx.doi.org/10.15405/epsbs.2018.03.3

Lyakh, V., Mikołajec, K., Bujas, P., Witkowski, Z., Zając, T., Litkowycz, R., \& Banyś, D. (2016). Periodization in team sport games: A review of current knowledge and modern trends in competitive sports. Journal of Human Kinetics, 54, 173-180. https://doi.org/10.1515/hukin-2016-0053

Murgu, A.-I. (2006). Fencing. Physical Medicine and Rehabilitation Clinics of North America, 17(3), 725-736. https://doi.org/10.1016/j.pmr.2006.05.008

Predoiu, R., Mitrache, G., \& Predoiu, A. (2017). The effects of fear, sadness and positive emotions on working memory in the case of female athletes. Romanian Journal of Experimental Applied Psychology, 8(1), 122-129. DOI: 10.15303/rjeap.2017.si1.a1

Roi, G. S., \& Bianchedi, D. (2008). The science of fencing: Implications for performance and injury prevention. Sports Medicine, 38(6), 465-481. https://doi.org/10.2165/00007256-200838060-00003

Terret, T., \& Ottogalli-Mazzacavallo, C. (2012). Women in Weapon Land: The rise of international women's fencing. The International Journal of the History of Sport, 29(2), 286-301. https://doi.org/10.1080/09523367.2012.641224

Turner, A., James, N., Dimitriou, L., Greenhalgh, A., Moody, J., Fulcher, D., Mias, E., \& Kilduff, L. (2014). Determinants of Olympic fencing performance and implications for strength and conditioning training. Journal of Strength and Conditioning Research, 28(10), 3001-3011. https://doi.org/10.1519/jsc.0000000000000478

Zadorozhna, O., Briskin, Y., Perederiy, A., Pityn, M., \& Sydorko, O. (2018a). Team composition in epee fencing which accounts for sportsmen's individual performance. Journal of Physical Education and Sport, 18(Suppl 4), 1863-1870.

DOI: 10.7752/jpes.2018.s4273

Zadorozhna, O. R., Briskin, Y., Perederiy, A., Pityn, M., \& Stepanchenko, I. N. (2018b). Improving fencers' theoretical training based on the stage reached in their basic development. IDO MOVEMENT FOR CULTURE. Journal of Martial Arts Anthropology, 18(2), 43-47. DOI: 10.14589/ido.18.2.6 